\section{Allergy Immunology}

\title{
Associate Editor Holger Garn
}

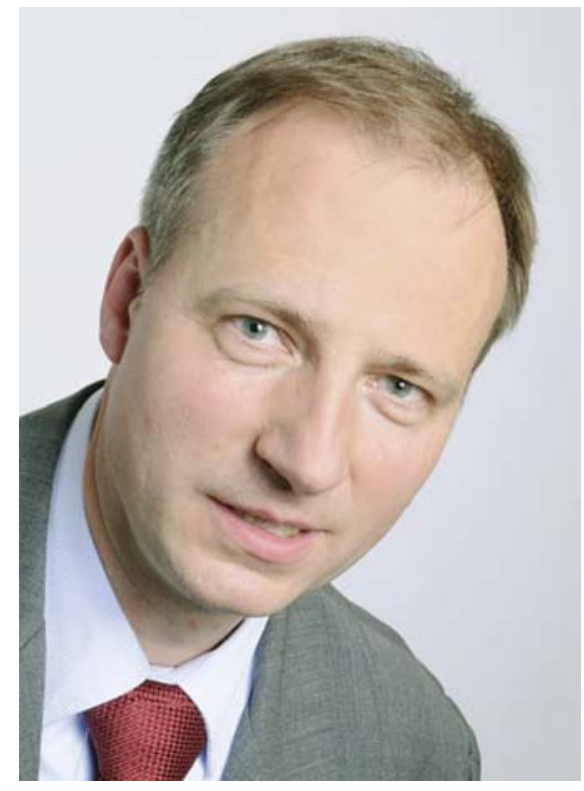

Holger Garn, Marburg.
Dr. Holger Garn, PhD, is Head of the Research Unit of the Institute of Laboratory Medicine and Pathobiochemistry, Molecular Diagnostics at the Medical Faculty of the Philipps University of Marburg, Germany. He studied Biology with a strong focus on physiology and immunobiology at the University of Leipzig, Germany, where he also earned his doctor's degree. After finishing his thesis, he went to Marburg and got a postdoctoral position at the Institute of Immunology of the Philipps University. He continued there as a project and work group leader, and in 2003, he assumed his current position. $\mathrm{He}$ is the cofounder of and served as managing director for Sterna Biologicals, a Marburg-based spin-off company that develops novel treatment options for chronic inflammatory disorders, including allergic diseases of the lung and skin.

His scientific interests are the development and characterization of animal models of acute and chronic inflammatory allergic disease conditions of the lung and the investigation of disease-causing pathomechanisms, with particular focus on the role of $\mathrm{T}$ cell subpopulations and their activation. Based on novel insights from epidemiological studies, Dr. Garn is also involved in the development of allergy-preventive strategies by early-life microbial exposure and the elucidation of the underlying immunological mechanisms of these approaches. Moreover, he develops novel antisense (DNAzyme)-based treatments for chronic inflammatory diseases from proof-of-concept in animal models towards human application.

Dr. Garn is a member of a variety of academic research organizations and societies. He regularly publishes peer-reviewed original manuscripts as well as review articles in leading journals in the field of allergy and immunology ( $>80$ publications), and provides lectures and presentations at renowned national and international scientific conferences. Additionally, he serves as a reviewer/associate editor for various leading journals in the field. 